\title{
Capacidade de vôo de quatro espécies de Charadriiformes (Aves) capturadas em Pernambuco, Brasil
}

\author{
Severino Mendes de Azevedo-Júnior ${ }^{1,3}$ \\ Manoel Martins Dias Filho ${ }^{2}$ \\ Maria Eduarda Lacerda de Larrazábal ${ }^{3}$ \\ Clyton José Galamba Fernandes ${ }^{3}$
}

\begin{abstract}
Flight range of Charadriidae and Scolopacidae trapped in Pernambuco State, Brazil. The northern coast of Pernambuco State, Brazil, is an wintering area for shorebirds originally from north America, especially from the east. The first flocks arrive in September, staying till April, when they molts, change plumage, and acquire enough biomass to migrate back to the northern hemisfere. In order to calculate flight range mass and length of the wing of Charadrius semipalmatus Bonaparte, 1825 , Calidris fuscicollis Vieillot, 1819, C. pusilla Linnaeus, 1766 and C. alba Pallas, 1764 was recorded. Traps were carried out in Coroa do Avião ( $7^{\circ} 40^{\prime} \mathrm{S}$ and $34^{\circ} 50^{\prime} \mathrm{W}$ ) in the northern coast of the State of Pernambuco, between May 1992 and April 1995. The length of the wing was registered with the wing spread (from the insertion of the bone till the extremity of the longest primary remige). Individuals which were changing the tenth primary were not included in this measuring. Mass was taken in grams, and flight range was calculated using the equations proposed for adult individuals. The individual with the highest flight range reached around $1831,87 \mathrm{~km}$, and had been trapped and ringed in $14^{\text {th }}$ April 1994, when had $61,5 \mathrm{~g}$ and wing measuring $12,9 \mathrm{~cm}$, with intermediate plumage. The highest flight range reached by $C$. fuscicollis was around $1906,43 \mathrm{~km}$. It had been trapped and ringed in the $3^{\text {rd }}$ of May 1992, with weight of 50 $\mathrm{g}$, and wing of $12,9 \mathrm{~cm}$, and had reproduction plumage. The individual with the highest flight range reached $3545,77 \mathrm{~km}$, had been trapped and ringed in $15^{\text {th }}$ April 1994 , weighted $44 \mathrm{~g}$, wing measured $10 \mathrm{~cm}$, and presented intermediate plumage. The specimen of $C$. alba which presented the highest flight range, of $82 \mathrm{~g}$, wing of 13,4 $\mathrm{cm}$, and had intermediate plumage.
\end{abstract}

KEY WORDS. Wing, mass, flight range, shorebids

O litoral Norte de Pernambuco constitui área de invernada para maçaricos e batuíras originários, sobretudo, do Leste da América do Norte. Os primeiros bandos chegam em setembro, permanecendo até abril, onde realizam a troca de plumagem e adquirem massa suficiente para as migrações de retorno ao hemisfério Norte (AZEVEDO-JÚNIOR \& LARRAZÁBAL 1999; AZEVEDO-JÚNIOR et al. 2001).

Em diversas espécies de aves limícolas, os dados coletados no campo, a exemplo de massa corpórea (peso fresco) e do comprimento da asa, permitem calcular a capacidade teórica de vôo, informações essas utilizadas no conhecimento das migrações intercontinentais.

1) Departamento de Biologia, Área de Zoologia, Universidade Federal Rural de Pernambuco. Rua Dom Manuel de Medeiros, Dois Irmãos, 52171-900 Recife, Brasil.

E-mail: smaj@npd.ufpe.br

2) Departamento de Ecologia e Biologia Evolutiva, Universidade de São Carlos. Via Washington Luiz, Km 235, Caixa Postal 676, 13565-905 São Carlos, São Paulo, Brasil. 
No Brasil poucos trabalhos foram desenvolvidos com o objetivo de estimar a capacidade de vôo de aves migratórias, dentre eles destacam-se estudos realizados com Calidris pusilla Linnaeus, 1766 no Parque Nacional da Lagoa do Peixe no Rio Grande do Sul (LARA-REZENDE et al. 1989) e no litoral do Maranhão e Pernambuco (ANTAS \& NASCIMENTO 1990; AZEVEDO-JÚNIOR \& LARRAZÁBAL 1999).

Com o objetivo de calcular a capacidade de vôo, foram coletados dados sobre a massa e o comprimento da asa de Charadrius semipalmatus Bonaparte, 1825, Calidris fuscicollis Vieillot, 1819, C. pusilla e C. alba Pallas, 1764.

\section{MATERIAL E MÉTODOS}

As capturas foram realizadas na Coroa do Avião ( $7^{\circ} 40^{\prime} \mathrm{S}$ e $34^{\circ} 50^{\prime} \mathrm{W}$ ), parte integrante do canal de Santa Cruz, litoral Norte de Pernambuco. Foram utilizadas dez redes ornitológicas (redes de neblina) com malha de $36 \mathrm{~mm}$ e $12 \mathrm{~m}$ de comprimento, no período de maio de 1992 a abril de 1995.

O comprimento da asa foi tomado com a asa esticada, desde o encontro dos ossos até a extremidade da rêmige primária mais longa (PRATER et al. 1977). Não foram consideradas para esta medição os indivíduos que estavam mudando a décima primária. A massa corpórea (peso) foi obtida com a utilização de balanças (pesola de 100 e 300), com unidade grama. Os indivíduos foram colocados em sacos de tecido de algodão para aferição da massa, subtraindo-se o valor do saco.

Para os cálculos da capacidade de vôo foram utilizadas as equações propostas por MCNEIL \& CADIEUX (1972b) para aves adultas. A capacidade de vôo em milhas/hora (FR) pode ser calculada através da equação: $\mathrm{FR}=\mathrm{F}$ x S x 9,1 kcal/FM; onde, F é o peso de gordura em gramas e $\mathrm{S}$ é a velocidade de vôo em milhas por hora, sendo de 35 milhas para Charadrius semipalmatus, de 50 milhas para Calidris fuscicollis e Calidris pusilla, e de 40 milhas para Calidris alba (MCNEIL \& CADIEUX 1972b). O fator 9,1 de multiplicação na equação acima, advém do fato de que a gordura tem uma densidade calórica média de $9,1 \mathrm{kcal} / \mathrm{g}$ (JOHNSTON 1970). FM é o metabolismo de vôo em kcal/hora e pode ser estimado através da equação: log $\mathrm{FM}=\log 37,152+0,744 \times \log \mathrm{W} \pm 0,074$; onde: $\mathrm{W}$ é o peso fresco em quilogramas de acordo com RAVELING \& LEFERVRE (1967). Segundo a equação proposta por MCNEIL \& CADIEUX (1972b), o cálculo de FR exige o conhecimento do peso de gordura $(\mathrm{F})$ em gramas que pode ser obtido indiretamente através da diferença dada por: $\mathrm{F}=\mathrm{FW}-y$; onde $y$ é o peso sem gordura em gramas e que pode ser estimado através de equação do tipo $y=\mathrm{a}+\mathrm{b} x$ onde $x$ é o comprimento da asa em centímetros. MCNEIL \& CADIEUX (1972b) observaram existir uma correlação significativa entre o peso $(y)$ e comprimento da asa $(x)$ para as algumas espécies de aves migratórias, dentre as quais, C. semipalmatus, C. fuscicollis, C. pusilla, e C. alba. Para as espécies estudadas o estimador para $y$ é dado pelas equações: $y=6,90+2,94 x$ para C. semipalmatus; $y=5,66+2,63 x$ para $C$. fuscicollis; $y=-7,01+3,34 x$ para $C$. pusilla e; $y=-42,49+7,81 x$ para C. Alba.

Desta forma, a equação original para FR pode ser transformada em: $\mathrm{FR}=$ $[(\mathrm{FW}-(\mathrm{a}+\mathrm{b} x)) \mathrm{S} 9,1 \mathrm{Kcal}][\text { Antilog }(\log 37,152+0,744 \log (\mathrm{W} \pm 0,074))]^{-1}$; fazendo com que a capacidade de vôo possa ser estimada a partir do peso fresco e do comprimento da asa aferidos em campo. Os valores das capacidades de vôo 
apresentados, foram originalmente calculados em milhas, sendo convertidos em quilômetros no momento da sua apresentação ( 1 milha $=1,60935 \mathrm{~km}$ ). Quanto ao comprimento da asa os valores foram convertidos de milímetros para centímetros.

Os resultados das estimativas de capacidade de vôo das espécies $C$. semipalmatus, $C$. fuscicollis, $C$. pusilla, e $C$. alba foram registrados utilizando-se gráficos de dispersão, onde cada ponto marcado representa um indivíduo capturado. Isto é, cada ave está representada por duas coordenadas: o comprimento de sua asa em centímetros $(x)$, e seu peso fresco em gramas (FW). Foram utilizados gráficos bidimensionais onde linhas de capacidade de vôo constantes foram traçadas. Os pontos de uma mesma linha de vôo constante, representam aves hipotéticas com diferentes comprimentos de asas e diferentes pesos. Estes pontos foram calculados a partir da equação anterior, que estima a capacidade de vôo (FR) de tal forma, que o comprimento da asa $(x)$ possa ser estimado, dada a capacidade de vôo desejada (FR) e um peso fresco qualquer (FW), onde: $x=[(F W-a)-F R($ Antilog $(\log 37,152+0,744 \log \mathrm{W} \pm 0,074))] b^{-1}$.

\section{RESULTADOS}

Das quatro espécies de Charadriiformes capturadas na Coroa do Avião foram aferidas as massas, comprimento de asa, médias e desvio padrão, cujos resultados encontram-se na tabela I.

O exemplar de $C$. semipalmatus que apresentou a maior capacidade de vôo estimada poderia atingir cerca de $1831,87 \mathrm{~km}$, foi capturado e anilhado em 14 de abril de 1994 com o peso de $61,5 \mathrm{~g}$ e a asa de $12,9 \mathrm{~cm}$, apresentando a plumagem intermediária.

O indivíduo de C. fuscicollis que apresentou a maior capacidade de vôo estimada poderia atingir cerca de $1906,43 \mathrm{~km}$, foi capturado e anilhado em 3 de maio de 1992 com o peso de $50 \mathrm{~g}$ e a asa de $12,9 \mathrm{~cm}$, apresentando a plumagem de reprodução.

O exemplar de C. pusilla que apresentou a maior capacidade de vôo estimada poderia atingir cerca de $3545,77 \mathrm{~km}$, foi capturado e anilhado em 15 de abril-de 1994 com o peso de $44 \mathrm{~g}$ e a de asa de $10,0 \mathrm{~cm}$, apresentando a plumagem intermediária.

$\mathrm{O}$ indivíduo de $C$. alba que apresentou a maior capacidade de vôo, atingiu cerca de 2010,70 km, foi capturado e anilhado em 28 de abril de 1995 com o peso de $82 \mathrm{~g}$ e a asa de $13,4 \mathrm{~cm}$, apresentando a plumagem intermediária.

As figuras de 1 a 12 relacionam os pesos, os comprimentos das asas, e as capacidades de vôo de indivíduos de $C$. semipalmatus, C. fuscicollis, $C$. pusilla e C. alba capturados em Pernambuco.

Tabela I. Massa corpórea (peso) e comprimento de asa de Charadriiformes capturados na Coroa do Avião, Pernambuco, de maio de 1992 a abril de 1995. (n) Número de indivíduos analisados.

\begin{tabular}{|c|c|c|c|c|c|c|c|c|c|}
\hline \multirow[t]{2}{*}{ Espécie } & \multirow[t]{2}{*}{$\mathrm{n}$} & \multicolumn{4}{|c|}{ Massa corpórea (g) } & \multicolumn{4}{|c|}{ Comprimento da asa $(\mathrm{cm})$} \\
\hline & & Máxima & Minima & Média & Desvio padrāo & Máxima & Mínima & Média & Desvio padrão \\
\hline Calidris fuscicollis & 5 & 50,00 & 32,00 & 40,70 & 5,87 & 13,00 & 12,30 & 12,58 & 0,30 \\
\hline Calidris pusilla & 229 & 46,00 & 18,00 & 27,09 & 4,80 & 11,00 & 8,70 & 9,98 & 0,32 \\
\hline Calidris Alba & 33 & 82,00 & 32,00 & 52,38 & 10,02 & 13,80 & 12,10 & 12,82 & 0,37 \\
\hline
\end{tabular}



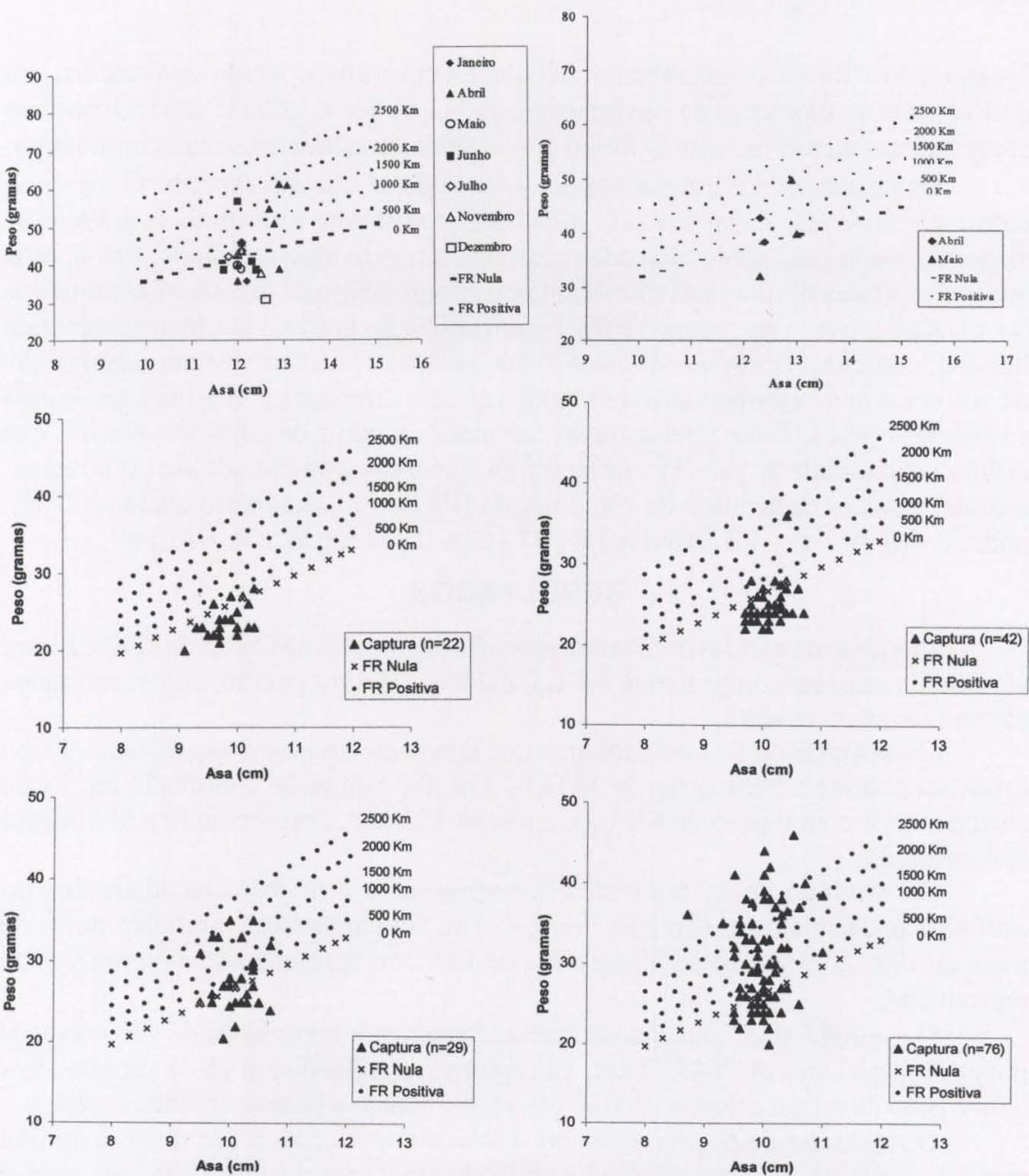

Figs 1-6. Capacidade de vôo de Charadriiformes adultos, capturados na Coroa do Avião, Pernambuco, de agosto de 1993 a abril de 1995. (1) Charadrius semipalmatus ( $n=21)$; (2) Calidris fuscicollis $(n=5)$; (3)Calidris pusilla $(n=22)$; (4) Calidris pusilla $(n=42)$; (5) Calidris pusilla $(n=29)$; $(6)$ Calidris pusilla $(n=76)$.

\section{DISCUSSÃO}

O peso e o comprimento da asa são fatores fundamentais na migração de caradrídeos e escolopacídeos. A capacidade de vôo pode ser analisada a partir do conteúdo de gordura e peso seco para algumas espécies de maçaricos e batuíras, permitindo assim, levantar informações sobre seus movimentos sazonais (MCNEIL 1969; MCNEIL \& CADIEUX 1972a).

O gasto de energia de aves limícolas pode estar mais baixo quando elas usam os ventos de cauda durante suas migrações. Um $C$. pusilla anilhado na costa de

Revta bras. Zool. 19 (Supl. 1): 183 - 189, 2002 

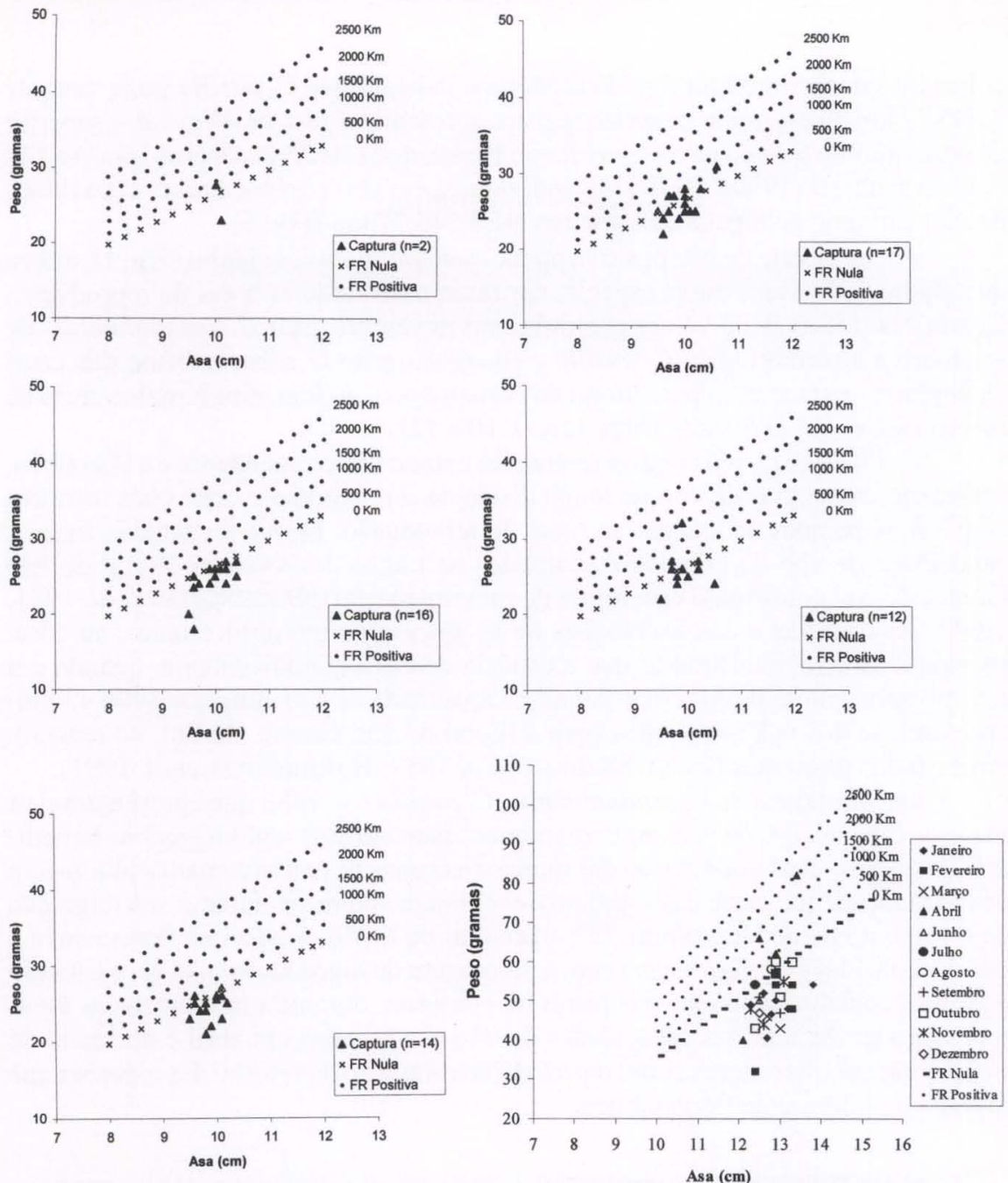

Figs 7-12. Capacidade de vôo de Calidris pusilla adultos, capturados na Coroa do Avião, Pernambuco, de agosto de 1993 a abril de 1995. (7) $n=2$; (8) $n=17$; (9) $n=16$; (10) $n=12$; (11) $n=14 ;(12) n=33$.

Maine (EUA), com $27 \mathrm{~g}$ foi recuperado na costa da Guiana dois dias depois (DUNN et al. 1988). BUTLER et al. (1997) conçluíram que os ventos de cauda são necessários para uma bem sucedida migração de primavera em Calidris mauri. O vento foi incorporado recentemente na teoria de uma migração mais favorável (WEBER et al. 1998). Todas as aves migratórias são beneficiadas pelo uso de ventos de cauda, no entanto, a migração ocorre também com ventos desfavoráveis (HEDENSTROM \& WEBER 1999). LARA-REZENDE et al. (1989) aplicando a fórmula de capacidade de vôo de MCNEIL \& CADIEUX (1972b) em C. pusilla, com os maiores pesos na Lagoa do Peixe, Rio Grande do Sul, obtiveram como estimativa de vôo 4.000 km. Dados 
coletados em abril no litoral de Pernambuco indicam que $C$. pusilla pode voar até $3.545,77 \mathrm{~km}$. O exemplar com este registro apresentava peso de $44 \mathrm{~g}$, valor superior ao peso mínimo teórico para $C$. pusilla no Brasil, que é de $25 \mathrm{~g}$, sugerido por ANTAS \& NASCIMENTO (1990). Os demais indivíduos, em abril, apresentaram capacidade de vôo variando de alguns quilômetros até 3.545,77 km (Fig. 6).

A capacidade de vôo positiva para C. semipalmatus em junho (Fig. 1), indica que alguns indivíduos dessa espécie, retornam mais tarde às áreas de reprodução. Condições positivas de vôo encontradas em novembro para C. semipalmatus, de setembro a novembro para $C$. pusilla e em agosto para C. alba, indicam que estes exemplares, ao passarem pelo litoral de Pernambuco, podem atingir outras áreas de invernadas ao sul do Estado (Figs 1, 8, 9, 10 e 12).

Calidris fuscicollis realiza migrações entre o Ártico canadense e a Patagônia, utilizando estratégias de vôo de longa distância sem paradas e com vôos menores com várias paradas até chegar ao local de reprodução. Dados levantados sobre a capacidade de vôo de indivíduos marcados na Lagoa do Peixe, no litoral do Rio Grande do Sul confirmam esta forma de movimentação (HARRINGTON et al. 1991, 1996). As capturas e as observações de $C$. fuscicollis em abril e maio, na costa pernambucana, demonstraram que a espécie ocorre esporadicamente, quando em retorno para o norte da América. A maior capacidade de vôo atingida (1906,43km) aproxima-se dos valores obtidos para o litoral do Rio Grande do Sul, no entanto, em períodos diferentes (LARA-REZENDE et al.1989; HARRINGTON et al. 1991).

Os indivíduos de $C$. semipalmatus, $C$. pusilla e $C$. alba que apresentaram as maiores capacidades de vôo em Pernambuco, estavam com a plumagem intermediária faltando apenas a conclusão das mudas das penas de contorno para a plumagem de reprodução, que neste caso será provavelmente adquirida durante sua migração de retorno ao hemisfério Norte. Já o exemplar de $C$. fuscicollis que apresentava a maior capacidade de vôo, estava com a plumagem de reprodução, não necessitando portanto, concluir as mudas das penas de contorno, durante seu regresso às áreas reprodutivas. As maiores capacidades de vôo encontradas em abril e no início de maio, indicam estes meses como o período da migração de retorno das espécies que invernam no litoral de Pernambuco.

AGRADECIMENTOS. Às Instituições: Universidade Federal de Pernambuco e Universidade Federal Rural de Pernambuco pelo apoio aos trabalhos de campo. Ao Prof. Dr. Antônio Rossano Mendes Pontes (UFPE) pela elaboração do Abstract.

\section{REFERÊNCIAS BIBLIOGRÁFICAS}

Antas, P.T.Z. \& I.L.S. Nascimento. 1990. Análise dos dados de anilhamento de Calidris pusilla no Brasil. An. Encontro Nac. Anilhadores Aves, Recife, 4: 6-12.

AZEVEDO-JÚNIOR, S.M. DE \& M.E. LARRAZÁBAL. 1999. Captura e anilhamento de Calidris pusilla (Scolopacidae) na costa de Pernambuco. Ararajuba 7 (2): 63-69.

AZEVEDO-JÚNIOR, S.M. DE; M.M. DiAs; M.E. LARRAZÁBAL; W.R.TELINO-JÚNIOR; R.M. LYRA-NEVES \& C.J.G. Fernandes. 2001. Recapturas e recuperações de aves migratórias no litoral de Pernambuco, Brasil. Ararajuba 9 (1): 33-42.

Butler, R.W.; T.D. Williams; N. WARnOCK \& M.A. BishoP. 1997. Wind assistance: A requirement for migration of shorebirds? Auk 114 (3): 456-466.

Revta bras. Zool. 19 (Supl. 1): 183 - 189, 2002 
Dunn, P.O.; T.A. MaY \& M.A. McCollough. 1988. Length stay and fat content of migrant semipalmated sandpipers in eastern maine. Condor 90: 824-835.

Harrington, B.A.; F.J. Leeuwenberg; S. Lara-Resende; R. McNeil; B.T. Thomas; J.S. Grear \& F. MARTINEZ. 1991. Migration and mass change of white-rumped sandpipers in North and South America. Wilson Bull 103 (4): 621-636.

- (1996) Migration and mass change of Calidris fuscicollis in North and South America. International Wader Studies 8: 136.

Hedenstrom, A. \& T.P. Weber. 1999. Gone with the wind ? A comment on Butler et al. (1997). Auk 116 (2): 560-562.

Johnston, D.W. 1970. Caloric density of avian adipose tissue. Comp. Biochem. Physiol. 34: 827-832.

LARA-ReZende, S. DE M.; F. Leeuwemberg \& B.A. Harrington. 1989. Biometrics of semipalmated sandpipers Calidris pusilla isouthern Brazil. Wader Study Group 55: 25-26.

MCNEIL, R. 1969. La determination du contenu lipidique et de la capacité de vol chez quelques espèces d'oiseaux de rivage (Charadriidae et Scolopacidae). Can. Jour. Zool. 47: 525-536.

MCNEIL, R. \& F. CADIEUX. 1972a. Fat content and flight-range capabilities of some adult spring and fall migrant North American shorebirds in relation to migration routes on the Atlantic coast. Nat. Can. 99: 589-605.

1972b. Numerical formulae to estimate flight range of some North American shorebirds from fresh weigth and wing length. Bird Banding 43 (2):107-113.

Prater, T. \& J. Marchant. 1977. Guide to identification and ageing of Holarctic Waders. 17. Tring. British Trust for Ornithology, 168p.

RAVELING, D.G. \& E.A. LeFERVRE. 1967. Energy metabolism and theoretical flight range of birds. Bird Banding 38: 97-113.

Weber, T.P.; T. Alerstam \& A. Hedenstrom. 1998. Stopover decisions under wind influence. Jour. Avian Biol. 29: 552-560.

Recebido em 19.X.2001; aceito em 25.VI.2002. 